\title{
Anthropometry in physical performance and health.
}

\author{
STEWART, A. and ACKLAND, T.
}

2018

This is an Accepted Manuscript of a book chapter published by Routledge/CRC Press in Body Composition: Health and Performance in Exercise and Sport on 6/6/2017, available online:

http://www.crcpress.com/9781351260008-6 


\section{Anthropometry in physical performance and health}

\section{Arthur Stewart ${ }^{1}$ and Tim Ackland ${ }^{2}$}

\footnotetext{
${ }^{1}$ Faculty of Health and Social Care, Robert Gordon University, Aberdeen AB10 7GJ, UK

${ }^{2}$ School of Sport Science, Exercise \& Health, University of Western Australia, M408, Perth 6009, Australia
}

\subsection{Introduction and Definitions}

\subsection{Overview of the physical bases of measurement}

\subsection{Seminal contributions to the field of kinanthropometry}

6.3.1 Matiegka's anatomical prediction of body composition

6.3.2 Lindsay Carter - somatotype, critical skinfold zone and more

6.3.3 The development of validated prediction equations for body composition

6.3.4 The standardisation of anthropometry and the emergence of International Society for the Advancement of Kinanthropometry

6.3.5 The advent of 3D photonic scanning

6.3.6 The International Olympic Committee ad hoc working group on body composition, health and performance

\subsection{Error control in anthropometry}




\subsection{Applications in health}

\subsection{Applications in sport}

\subsection{Applications in ergonomics}

\subsection{Conclusion}

\subsection{Introduction and definitions}

Anthropometry is defined as "The scientific procedures and processes of acquiring surface anatomical dimensional measurements such as lengths, breadths, girths and skinfolds of the human body by means of specialist equipment” (Stewart, 2010). This approach has altered little if at all over the last hundred years, and even in ancient Greece, we hear of systematic body measurement in order to produce statues which were appropriately sized to real individuals. Sculptors would have appreciated that this approach demands painstaking detail, adherence to best practice and diligence in reducing errors, and few scientists would argue with this. Anthropometry sits within the field of kinanthropometry - "The academic discipline which involves the use of anthropometric measures in relation to other scientific parameters and/or thematic areas such as human movement, physiology or applied health sciences” (Stewart, 2010). However, one of the issues for kinanthropometry, particularly in its applications for physical activity and sport, is that the tools have not advanced in parallel with those of other disciplines such as sports physiology and biomechanics. Researchers, therefore, may be persuaded to think that its relevance is reducing in a contemporary research context. Indeed, for publications in two main research journals, the prevalence of anthropometry as central to research (estimated from key word searches using similar terms) appears to have peaked a generation ago (Olds, 2004). But perhaps kinanthropometry is on the verge of a renaissance for two reasons. Firstly, the field has now largely embraced tightly defined standard procedures and error control, the lack of which previously diminished its 
ability to convince a research community becoming accustomed to more sophisticated methods. Secondly, recent advances in digital anthropometry, using 3D body scanning, enable an unprecedented range of new measurement possibilities. These new measures can augment traditional anthropometry, and the combination of manual and digital anthropometry may allow new research questions to be addressed.

\subsection{Overview of the physical bases of measurement}

\subsubsection{Skinfolds}

The skinfold is a manually held, compressed layer of subcutaneous adipose tissue plus skin. Most calipers seek to provide a constant compressive force of $10 \mathrm{~g} \bullet \mathrm{mm}^{-2}$ at all jaw opening distances. While validated against other measurements for fat quantity, several limitations have come to light from research evidence which are worthy of note. Firstly, adipose tissue and the fat within it are compressible with a small applied force, and the compressibility curve of skinfolds varies both within and between individuals. This underscores the importance of rigour when locating skinfold sites (Hume and Marfell-Jones, 2008), caliper orientation and other aspects of technique (Stewart et al., 2011), and the time of compression (Himes, Roche and Siervogel, 1979). Different quantities of connective tissue structures embed the adipose tissue, meaning that skinfolds of a given magnitude represent a highly variable quantity of fat between individuals.

\subsubsection{Girths}

Girths represent curvilinear distances around a body segment. As with skinfolds, their measured value will differ markedly if the measurement is made in a slightly different location (Daniell, Olds and Tomkinson, 2010). Referred to as circumferences, they are not strictly circular but rather, irregular and at best elliptical, with the extent of non-circularity varying regionally and by overall body size. Empirical logic suggests girths may also be 
affected by hydration status, if significant water loss occurs from fluid compartments, and other circulatory responses do not compensate. In the case of thigh girth, for example, glycogen together with its bound water molecules can increase the girth value, if exercise or dietary intervention has perturbed levels prior to measurement. Metal tapes used to measure girths should not compress the skin surface, and close inspection of the girth should reveal no indentation of the skin (Stewart et al., 2011). In practice this is straightforward if the anthropometrist's fingers are used to pin the tape against the skin surface without compressing it. Girths should also span concavities - such as the lower back when measuring the waist. This does make girths measured by tape shorter than the equivalent surface distance -this may be an important distinction with three-dimensional (3D) scanning which generally identifies the surface distance. Tapes which are made of cloth may stretch and generally lack sufficient rigidity for easy measurement, and all tapes should have a stub beyond the zero value which can be held by the measurer.

\subsubsection{Segment lengths, skeletal breadths and anatomical heights}

Linear distances between points on the body surface, or from the standing or sitting surface to key landmarks, provide valuable information of the body’s proportions. The approach requires that landmarks can be located visually and in a systematic manner. The majority of landmarks are identified from the underlying skeletal structure, such as the anterior superior iliac spine (ASIS) and acromiale. Others are identified from soft tissue structures, such as the omphalion (midpoint of the naval) and tragion (the notch structure in the outer ear). Bony landmarks require palpation and it is paramount that these are marked once the skin has returned to its ‘resting orientation'. In addition, tight clothing being moved to facilitate the marking may displace the skin surface by several centimetres, so care is required to ensure such marks are made appropriately. Segment lengths and heights are usually measured using a segmometer, large sliding caliper or traditional rod anthropometer. Anatomical heights are 
best measured to an anthropometric box, rather than the floor, for ease of measurement. Care is required when placing the instrument on the landmarks to ensure correct alignment, to check for slippage and to avoid compression of the skin. For skeletal breadths, compression of the overlying tissue is required, demanding a firm grip so the soft tissue contributes only minimally to the recorded measure. This means traditional anthropometric measurements are not compatible with 3D scanning, in which breathing and postural artefacts can affect some measurements.

\subsubsection{Volumes and areas}

Three dimensional photonic scanning creates a digital shell of the body which can be interrogated for such measurements as surface distance, cross sectional area, surface area, and segmental and total volumes. Certain mathematical assumptions are necessary with the different approaches to geometric calculations for body shape. Methods for acquiring scans vary, but typical ‘booth’ scanners have footprints to guide participants when orienting themselves appropriately. With portable scanners, it is useful to capture a horizontal floor section which can be used to locate and rotate an "xyz positioning tool”, which can then describe all landmarks and subsequent measurements in a standardised way. Identifying areas and segmental volumes vary by software options, with more primitive versions constraining sub-analyses to orthogonal axes. More sophisticated software can depict the body at any angle by defining a slice plane based on a minimum of three points. So for example, to replicate the waist girth or area, a landmark will be placed onto the torso and positioned to comply with the required waist protocol (Stewart et al., 2010). With more advanced software, tilting the waist plane is possible so that it could be made perpendicular to the torso, and thus comply with the minimum waist as identified by the International Society for the Advancement of Kinanthropometry (ISAK). 


\subsection{Seminal contributions to the field of kinanthropometry}

\subsubsection{Matiegka's anatomical prediction of body composition}

The first systematic body composition study was in 1921, when Jindrich Matiegka developed a validated method of quantifying bone, muscle and adipose tissue, together with residual mass using a geometric anthropometric approach (Matiegka, 1921). He applied this to quantify tissue components amongst different professional groups including barbers, blacksmiths, hairdressers and gymnastics instructors. Although his imperative was to examine the capacity of the body for physical work, which was an important consideration in the aftermath of World War I, Matiegka's approach spearheaded many other research efforts in body composition using anthropometry.

In the 1980s our understanding of the anatomical model championed by Matiegka was considerably advanced with the advent of the Brussels Cadaver Study. This project involved full anatomical dissection of 25 cadavers, with the separate tissue compartments fully dissected and weighed. The magnitude of this effort was enormous and perhaps only truly appreciated by those who have undertaken whole body dissections. The multiple anthropometric measurements made on the cadavers greatly enhanced our understanding of the nature of the skinfold (Clarys, Martin, Drinkwater and Marfell-Jones, 1987) and enabled the estimation of muscle mass (Martin, Spenst, Drinkwater and Clarys, 1990) and skeletal mass (Drinkwater and Ross, 1980).

\subsubsection{Lindsay Carter - somatotype, critical skinfold zone and more}

J.E. Lindsay Carter is perhaps best recognised as the protagonist of the Health-Carter somatotype method in 1967.This method effectively brought the physique analysis schema, collectively referred to as "the somatotype", into a convincing and utilitarian process which rated physique according to degrees of adiposity, musculoskeletal robustness and linearity. This concept was to prove more popular than any before or since, partly because it enabled 
anthropometrists with a basic training in 10 measurements to become somatotype raters. Later, the somatotype concept was extended to provide reference ranges for a large number of different sporting groups, children, the elderly and those of varying ethnicity (Carter and Heath, 1990).

During the Olympic Games in Mexico City in 1968 and Montreal in 1976, Dr Carter was instrumental in conducting systematic surveys of the Olympic athletes, reporting somatotypes and a range of other anatomical measurements. These landmark surveys would become some of the largest measurement endeavours ever undertaken, assessing nearly 2000 individuals in total. The breadth and depth of this athletic sample was unprecedented and is not likely to be repeated, due to greater logistical and security challenges facing measurers at public events. Dr Carter's data led him to espouse a 'critical skinfold zone', which identified the optimal and critical levels of skinfolds observed in athletes. This he related, subsequently, to a simple biomechanical model, firstly by suggesting that the human body comprises "productive mass” which contributes to movement (muscles, bone, nerve tissue, blood and essential regulatory organs) and "ballast” (excess fat) which adds to the cost of movement (Carter, 1985). Such an approach, while differing from contemporary understanding of adipose tissue as an endocrine organ (Kershaw and Flier, 2004), was based on a very large number of observations which have been replicated by others since, and represents the biomechanical imperatives which continue to inform conditioning approaches in sport.

\subsubsection{The development of validated prediction equations for body composition}

Over 100 prediction equations exist for adults, and specific subgroups by ethnicity, age, athletic status or medical condition. These use skinfold values and are modelled (e.g. using linear, logarithmic or quadratic equations) to predict fat as determined by another method, which has historically been underwater weighing. Some of the early generalised equations from the 1970s had very limited accuracy (standard error of the estimate $~ 5 \%$ fat, as 
determined by underwater weighing). In other words, if a person was measured at $15 \%$ fat, then the measurer could only be $68 \%$ confident of the true value falling between $10 \%$ and $20 \%$ fat, or $95 \%$ confident of the actual value falling between $5 \%$ and $25 \%$ fat. Furthermore, this fails to consider the possibility that the reference method itself may not be accurate, as Adams et al. (1982) demonstrated for underwater weighing in Canadian football players. Sinning et al. (1985) revealed that the vast majority of generalised fat prediction equations were of very limited use for assessing athletes because they have less fat and distribute it differently from non-athletes. Furthermore, the reference methods often required unsupportable assumptions such as the constant density of the fat-free mass (Martin et al., 1986). Most equations in common use during the latter part of the $20^{\text {th }}$ Century were of unacceptable accuracy for use with athletes, except for equations by Jackson and Pollock (1978) and Jackson et al. (1980). Crucially, these equations were validated as part of the original study, unlike those of Durnin and Womersley (1974) which by comparison, systematically overestimated fatness. This may be due, in part, to differences in the precise technique - descriptions of which left room for variable interpretation. In addition, Lohman (1992) observed that systematic differences of 3-4\% fat were obtained using different types of caliper.

Lohman had previously developed a robust procedure for cross validation of equations (Lohman, 1992). This outlined several key principles including reporting standard errors of the estimate $(\mathrm{SEE})$, total error $(\mathrm{TE})=\left[\sum(\text { reference method fat mass - predicted fat mass })^{2} / n\right]$, where $n$ is the number of participants, and fitting curvilinear lines to data. Perhaps more than any other individual, Lohman shaped the progress of kinanthropometry by this approach, together with steps towards standardisation of technique, which led to the Anthropometric Standardization Reference Manual (Lohman, Roche and Martorell, 1988). He also recognised that too many researchers were developing new equations, many of which were 
no better than existing ones. As a result, Lohman recommended combining data from different studies, altering intercepts from existing equations and only publishing new equations if they outperformed existing ones (Lohman, 1992).

One study which did fulfil these criteria was that of Stewart and Hannan (2000) who predicted fat mass and fat-free mass from skinfolds in 106 male athletes from a wide variety of sports. The authors used dual X-ray absorptiometry (DXA) as the reference method and achieved a standard error of the estimate of $1.7 \mathrm{~kg}$. This study utilised a stepwise regression method to select the optimal skinfolds from a total of 19 sites across different body regions, and concluded that appropriate skinfold selection is a crucial element in the accuracy of a prediction equation for a specific population. Using a random sub-sample of 82 to develop the equation and the remaining 24 to validate it, the following equation was produced: fat mass $(\mathrm{g})=331.5($ abdominal $)+356.2($ thigh $)+111.9 \mathrm{M}-9108$ $\left(\mathrm{R}^{2}=0.81\right.$; $\mathrm{SEE}=1732 \mathrm{~g}$; $\left.\mathrm{TE}=2.9 \%, P<0.001\right)$, where $\mathrm{M}$ is body mass in kg; skinfolds in $\mathrm{mm}$. With increased heterogeneity in a sample, the greater the scope for generalisation of an equation, but this leads to poorer accuracy (i.e. a greater standard error). Researchers need to balance the capability for an equation to apply to other groups, with a low error. However, equations with a low SEE may still not be effective for fat prediction; it is also important to consider total error (TE).

More recently, researchers have had access to four-compartment reference methods which separately divide the fat-free mass into bone mineral, water and other tissue. These components represent the best available criterion against which anthropometric predictions can be made, and contribute more valid equations for use in kinanthropometry. However, the costs for such investigations may limit the likelihood of them being repeated elsewhere. One example of excellent practice is the work of Evans et al. (2005) who studied collegiate athletes of both sexes and mixed ethnicity. Their resulting equation thus had separate gender 
and ethnicity terms in the regression, and yielded a prediction of body fat with a total error of 3.8\% - which is lower than the equations from Jackson and Pollock (1978) and Jackson et al. (1980) developed using underwater weighing alone. Interestingly, the final regression that used only three skinfolds (abdominal, thigh and triceps) had comparable accuracy as the one involving seven sites.

\subsubsection{The standardisation of anthropometry and the emergence of International Society for the Advancement of Kinanthropometry (ISAK)}

Historically, routine anthropometric measurements have been employed in the fields of nutrition, medicine, sport and ergonomics. Their use in diverse academic disciplines throughout multiple countries has led inevitably to different approaches, nomenclature and practices, which represented a barrier to progress in the fields of application.

ISAK was founded in Glasgow, UK at a conference in 1986 from interested practitioners in the field, and members of the previous international working group in kinanthropometry, seeking to standardise practice. In the same year, a consensus conference in Virginia, USA sought to do the same. Both were motivated by the same drivers - the need for better standardisation in measurement description, protocols and practice, and accuracy. Both were ultimately successful in achieving these aims, although their approaches differed. In USA during the 1980s the strong research base for clinical body composition covered a range of technologies, and anthropometry took its place alongside them. The standardisation manual (Lohman et al., 1988) was a culmination of previous research and was, therefore, less didactic and constraining than the ISAK scheme which was influenced more by the disciplines of sport, exercise science and human biology. By contrast, ISAK’s approach was to introduce a teaching and practice structure, which was subsequently manifest in a new qualifications scheme in 1996. It was heavily influenced by practitioners at the Australian Institute of Sport, with the first steps towards its definitive manual being chapter 2 of the Anthropometrica book 
(Norton and Olds, 1996) which systematically detailed protocols and applications of anthropometric measures in an accessible and unprecedented way. Comparing these two approaches reveals significant differences in perspective. The Lohman et al. (1988) manual provides a rich literature, strong evidence base, and relevant clinical detail, for instance in the different contribution of various superficial fat depots to health risk (p55). However, it suggests that the location of skinfold sites need not be marked on the participant as a general rule, making exceptions for inter-caliper differences, and the combination of skinfolds with girths to estimate limb cross sectional areas. This contrasts with the strict adherence to the protocol for marking landmarks using the ISAK method for skinfolds, which considers it fundamental to anthropometric measurement. Moreover, the actual marking itself defines the orientation and precise alignment of the index finder prior to the fold being raised. Perhaps the main reason for increased popularity of the ISAK approach is not its stance on land marks, but the adoption of a 4-level hierarchy of practitioner licencing, based on competence assessed by practical exam. Crucially, ISAK requires that all measurers be licenced, even the examiners, who are required to examine one another's measurements. Such a comparison requires all measurers to pass error control targets in terms of reproducibility in a standardised setting. This includes inter-measurer as well as intrameasurer reliability, and represents a great advance for the methodology. While other schemes might be able to demonstrate reproducibility within a single laboratory, the ISAK protocols provide an indication of the comparability with others from various laboratory settings. Indeed, where technique, instruments and protocols vary, there will inevitably be considerable inter-laboratory variability; discussion of which has been conspicuously absent from the publication record. In contrast, adhering rigidly to the same protocol and quantifying error enables data to be pooled between laboratories with more confidence, and reference ranges to be constructed. ISAK has also championed the adoption of using raw 
data scores as opposed to conversion into \% fat values, made possible only by strict protocol definitions and quality assurance of individual measures. In addition, the need for practitioners to have a measurement licence has fostered the automatic re-skilling of practitioners, and enabled a forum for scrutiny and practice of protocols and technique. Such scrutiny and feedback resulted in small but significant changes to the teaching manual, now in its $3^{\text {rd }}$ revision (Stewart et al., 2011). This manual has been translated into several languages for use across the globe. Even in the USA where body composition research has been strongest, and the Lohman et al. (1988) manual has received widespread acclaim and usage, ISAK as a concept is being adopted, albeit more slowly than elsewhere in the world. The ISAK approach is not the only way of performing manual anthropometric measurements, but it continues to flourish, and is used more than any other protocol with elite athletes (Meyer et al., 2013).

\subsubsection{The advent of three dimensional photonic scanning (3DPS)}

The advent of 3D photonic scanning has enriched the tools available for describing the body. Referred to in some circles as 'digital anthropometry', 3DPS has made a vast contribution to body measurement. It has the capability for measurements which include total volume, segmental volume, curved surface distances, direct distances, perpendicular planar distances, surface areas and cross sectional areas. Not only have these become valuable quantities in their own right, they have informed novel approaches to quantifying the body that are not possible with traditional anthropometry. The term 'scanning' is used by medicine and industry to acquire a vast array of measurements. In humans, 3DPS involves approaches to acquiring the body shape which most commonly include structured light, class 1 lasers and more recently, depth cameras.

Structured light is projected onto the body to produce patterns distorted by the surface contour. Similarly, class 1 (eye-safe) lasers can project onto the body and for both, digital 
cameras placed around the body detect the deformed light stripe against a 3D reference grid, and individual points are calculated via triangulation. More complex approaches, such as stereo photogrammetry and others, are necessary when using handheld scanners which are not constrained to horizontal array beams. Depth cameras, by contrast, acquire a single image and project an infra-red speckle pattern onto the participant, while the sensor captures its deformation. This deformation, together with accurate distance sensing and RGB colour enable a 3D image to be acquired. For single depth cameras, software approaches include libraries of images to estimate the 'blind side' data obscured from view. As yet, depth cameras lack the resolution and accuracy of structured light and laser scanners, although the technology is rapidly developing.

Once the primary measurement data are obtained in a point cloud, subsequent processing involving hole-filling and smoothing is done either manually or automatically via system software. In the case of hand held scanners, images require additional steps to register separate scan fragments, and then fusion into a single object. Analysis can be performed either by the user identifying specific measurement locations, or from locations which are automatically detected from the shape itself. These 'primary' landmarks frequently include the vertex, axilla and crotch and may be used to generate secondary landmarks where specific measurements are made. Landmarks may also be applied via affixed reflective dots or triangles on the skin surface. Alternatively, landmarks may be created digitally once the 3D scan has been rendered into an object file. Increasingly, computing approaches can derive landmarks automatically from edge detection or shape curvature algorithms with minimum user involvement. Lastly, there is also a case for using visible landmarks, such as the Adam's apple, axillary fold and naval, together with maxima and minima positions to describe the body. Some measurements are required to be made when posturally constrained, for example in disabled participants, or for ergonomics applications. Maximum and minimum values can 
contribute to our knowledge of measurement variability in humans, for example at the waist (Stewart et al., 2010).

Greater resolution of 3DPS systems means increasingly dense meshes to describe the body shape, at the expense of increasing file size. For example, early fixed position industrial scanners developed for the clothing industry towards the end of the 1990s acquired about 200,000 points, and subsequent refinement of scanners increased this three or four-fold. A typical adult scan from a Hamamatsu BLS 9036 (Hamamatsu Photonics, Hamamatsu, Japan) would have 700,000 vertices and a file size of 13MB. In contrast, a full body scan acquired by the portable Artec L scanner (Artec Group, Luxembourg) would have up to 20 million vertices with a corresponding file size of 500MB.

In conventional anthropometry, there have been attempts to standardise practice for well over a century, illustrating a genealogy of protocols used in sports science, clothing, ergonomics and health (Kupke and Olds, 2008). Their work continues this process into the realm of 3D scanning, and identifies the need for generalised language, dimensional syntax and the means to describe anthropometric functions. A project-specific land marking manual (Olds et al., 2004) provides better definitions and more information than other standards (BSI 2010; Robinette et al., 2002), yet the disparity in what constitutes a standard definition underscores the diversity of approach in this new and expanding field. Efforts to standardise 3DPS are still limited by the relatively few scanning facilities for research, together with the rapid advancement of this technology. However, there is a burgeoning interest in professional meetings for 3DPS and, with the establishment of professional networks, the steps to formalise and standardise practice are imminent.

Total body volume enables the calculation of body density, and thus an estimate of \%fat via the 2-compartment method. Important validation work was carried out by Wang et al. (2006), with a C9036 laser scanner (Hamamatsu Photonics, Hamamatsu, Japan) compared to 
underwater weighing and anthropometry, using both mannequin and a human sample with a wide age range (6-83 y). Significantly larger body volumes with scanning were found compared with underwater weighing. This may be attributable to the difficulty in exhaling completely while standing upright in the scanning pose, the variable density of the fat-free mass in the sample, or the possibility that excess body hair causes the scanner to identify a false surface in some instances. The use of the mannequin to compare manual girths and those assessed by 3DPS revealed that manual anthropometry yielded mean values which were $0.87 \%$ greater at the waist and $0.55 \%$ greater at the hip, but $0.66 \%$ lower at the thigh. This difference is within the measurement error anticipated from experienced anthropometrists and may reflect minor orientation or positional differences in measurement. This should give confidence in the capability of 3DPS to extract valid measurements. However, like conventional anthropometry, hip girths and female chest girths will always be made over clothing, and the use of suitable form-fitting apparel is essential, provided it does not compress the body shape appreciably.

While 3D scanning will never be able to replace conventional skinfolds and skeletal breadths (due to the compression required to locate the bony landmarks), it offers a range of measurement possibilities which augment and enhance conventional anthropometry. Firstly, there is the capability for retrospective measurement of a scan after a participant has left the laboratory, which can be subsequently interrogated for data - an approach used in telemedicine in remote regions of the world for medical data and investigations. Secondly, there is the opportunity for scan data to be sent elsewhere for analysis, using bespoke software for specialist purposes. Thirdly, there is the opportunity to create a template based approach for locating other measurements (e.g. skinfolds or ultrasound fat measurements) in xyz space in a composite model. Lastly, 3DPS allows the possibility of visualisation, for instance in body image assessment or somatotyping (Olds et al., 2013), which may extend to novel interfaces 
with interactive capabilities, rapid prototyping and the construction of figurines to depict individuals or averaged shapes of groups. Such exciting new terrain will undoubtedly extend the applications of anthropometry into uncharted territory.

\subsubsection{The International Olympic Committee (IOC) ad hoc working group on body composition, health and performance}

This working party met from 2010-2013 to address concerns related to the health and performance of athletes who attempt to modify their body composition using extreme and sometimes dangerous methods. This problem was of special concern for the weight sensitive sports; where athletes are required to make a weight category, or are judged on aesthetic criteria, or where body composition markedly affects performance. The group reviewed literature, conducted research and surveyed scientists, coaches and health professionals before disseminating their findings. Specifically, the aims and resulting outputs of this working party were as follows.

- To identify medical problems_due to unhealthy practices in sport leading to extremes of underweight, weight reduction and dehydration - see Sundgot-Borgen et al. (2013).

- To identify research needs in body composition, health and performance - see Ackland et al. (2012); Meyer et al. (2013); Müller et al. (2013a,b); Müller et al. (2016).

- To identify current practice for body composition assessment globally - see Meyer et al. (2013).

- To develop suggestions for practical strategies capable of solving body composition and underweight problems in sports - see Müller et al. (2006).

- To establish, if practicable, an optimum body composition and/or minimum weight values for healthy competition in sports - see Sundgot-Borgen et al. (2013). 
One of the important advances led by this group has been the development of an accurate and repeatable method for sampling the subcutaneous adipose tissue layer using ultrasound (Müller et al.,2016). The standard methodology has been published, together with data on accuracy and repeatability, so now the group is completing a multicentre trial to assess interand intra-tester reliability and to compare ultrasound results with DXA, skinfolds and a multicomponent model.

The ultrasound technique provides an accurate measure of the depth of the uncompressed subcutaneous adipose tissue (SAT) at several sites on the body surface. For several participants with extremely low fat content, measured during the multi-centre trial, revealed results which challenged conventional understanding relating to the fat content of skinfolds. One Caucasian athlete (soccer) with a skinfold total across the 8 ISAK sites of $31.9 \mathrm{~mm}$, had an uncompressed total fat depth of just $6.0 \mathrm{~mm}$ - only $19 \%$ of the skinfold total. One AfroCaribbean athlete (track and field) whose skinfold total was $36.9 \mathrm{~mm}$, had just $3.8 \mathrm{~mm}$ of total fat depth - only $10 \%$ of the skinfold total. Strikingly, significant connective tissue was apparent in the images of both, but more so in the track and field athlete. Both participants were apparently healthy and hydrated at the time of measurement. While these results require further replication in other centres, they challenge the concept of a minimum skinfold total for a certain amount of fatness in athletes, and cast doubt on the value of skinfolds to identify fat in extremely lean individuals.

\subsection{Error control in anthropometry}

Error in anthropometric measurement is inevitable. It relates both to the acquisition of the measurement, where equipment calibration, site location and technique are important, as well as the biological error, more correctly termed 'biovariability', in which the true value changes over time. Biovariability relates to diurnal variation, hydration levels, temperature and other metabolic processes. Errors associated with repeated measurements in anthropometry are 
quantified by the use of repeated measurements on the same testing occasion. Clearly, if measurements are made in rapid succession the true value should not have changed.

However, in the case of skinfolds, some residual compression may remain for up to 2 minutes, resulting from realignment of fat lobules in adipose tissue, so re-testing within this time is discouraged. When measuring a parameter for the second time, it is important that the measurer is blinded to the first measurement scores. Quantification of the error is commonly achieved by the intra class correlation coefficient and the technical error of measurement statistics.

The intra class correlation coefficient is a correlation between successive measurements made on the same subject, by a group of measurers. Although popular, it is sensitive to the sample mean and alternatively, the technical error of measurement (TEM) statistic, either as a raw score (absolute) or expressed as a percentage of the mean value (relative) may be preferable. The absolute TEM is calculated by the expression

$$
\mathrm{TEM}=\sqrt{\frac{\Sigma d^{2}}{2 n}}
$$

Where $\Sigma d^{2}$ is the sum of squared differences between measurements and $n$ is the number of participants measured. The absolute TEM is multiplied by 100 and divided by the 'variable average value' (overall mean of the means between measurements of each participant for the same site) to provide the relative TEM (\%TEM) for each site of measurement (Perini, de Oliveira, Ornellas and de Oliveira, 2005).

There exists a 1-2\% diurnal variation in stature as a consequence of gravitational forces acting on compressible structures within the spine when upright (Tyrell, Reilly and Troup, 1985). Coupled with the body’s normal eating and bowel activity, this translates to the derived body mass index varying by about $1 \mathrm{~kg} \bullet \mathrm{m}^{-2}$ per day for a typical adult, but considerably more where dehydration is also an issue, such as for athletes of certain sports. Gravity also influences fluid components of the body in other less rigid tissues, such that 
posture can influence body composition measurements. Cairn-Levy et al. (2008) demonstrated such a difference in standing and supine skinfold measures. This may not come as a surprise, but should be considered in certain patient groups. Extending the logic of this finding, we know remarkably little about how the plasticity of tissues under their own weight affect the comparability of standing and supine measurements using medical imaging, as well as anthropometry.

\subsection{Applications in health}

The categorisation of an individual's health risk based on simple anthropometric measurements has been a ubiquitous front line for health surveillance in medicine for over half a century. While this process assists in channelling resources to where they are most required, the candidate measures used to assess body size or relative weight also have the potential to mislead. This is a direct result not only of the variability in body composition and body proportions, but the variable susceptibility for disease between different individuals. Increasing use of clinical guidelines and pathways has forced different measurements to be made, by different individuals, at different stages of diagnostic and care pathways. Simple anthropometric measures such as stature, mass, waist and hip girth may engender a risk that the need for training and quality control is overlooked.

It is surprising, therefore, that the diagnosis of health risk could be based on measurements made by individuals whose error control may not have been properly appraised. As discussed in section 6.4, precise anatomical location of the measurement site is pivotally important. Skinfolds show significant variation by distance at all eight standard sites, but the magnitude and direction of the differences varied by site location (Hume and Marfell-Jones, 2008). Girths, while not appearing to be critical at the forearm and calf, demonstrate critical variation at the more commonly used waist and hip locations and shows slightly greater variation in females than males (Daniell, Olds and Tomkinson, 2010). 
Anthropometric measurements are capable of providing a full fractionation of body mass using an anatomical model based on cadaver dissection (Drinkwater and Ross, 1980). This expanded the original work by Matiegka (1921) including the prediction of skeletal, muscle, fat and residual masses. The methodology of subtracting skinfolds multiplied by pi from body segment girths was applied as part of this to generate a theoretical lean girth, and this approach was applied to predict falling in the elderly using calf girth (Stewart, Stewart and Reid, 2002). However, for most health purposes, such detailed measurement is not warranted. Nevertheless, some anthropometric measurements have a valuable role to play in health surveillance for appropriate weight and metabolic risk. In many cases, they may represent the first line of defence in flagging individuals for follow up or specific treatment either for undernourishment or excessive fatness. They represent convenient surrogates for health, at an appropriate level of technology and cost for screening initiatives.

The question of which measures are the most appropriate is a matter of heated debate between physicians, epidemiologists and scientists. A large array of direct measures, derived indices or composite indices are available from anthropometry, as illustrated in figure 6.1.

*** figure 6.1 near here $* * *$

Total body fatness might be the logical objective to address the obesity research agenda, not least because obesity is defined as an excess of bodily fat. However, there is neither clear linkage between fat levels and morbidity and mortality, nor recommended reference ranges of body fat which can be generalised with confidence (Gallagher et al., 2000). The metric for defining overweight and obesity - the body mass index (mass•stature ${ }^{-2}$ ) does have such reference ranges, but is at odds with the fundamental definition of obesity, assuming excess weight equates to excess fat. The BMI's critics point to the mis-classification of muscular individuals as overweight or obese, and the challenges of applying different cut-off values to classify different groups, while its supporters will cite that at a population level, more 
individuals are likely to be heavy due to an excess of fat than muscle. If the body fat content can be measured, or predicted from skinfolds, the fat-free and fat masses can be used independently to calculate separate indices. Another improvement over BMI is the mass index (Ackland et al., 2012) which is based on the cormic index (sitting height•stature ${ }^{-1}$ ) and accounts for relative leg length in its scoring. This index is used in rule setting for ski length in Olympic ski jumping.

If the focus is on metabolic health there is clear evidence that health risk relates more to centralised fat on the torso and abdomen, rather than total fat (Bjorntorp, 1997). While logic would suggest the waist circumference should thus inform us more of health risk than would the body mass index, it us unable to distinguish between subcutaneous (SAT) and visceral (VAT) adipose tissue compartments. Because visceral fat is an independent predictor of mortality (Kuk et al., 2006), for two individuals with a similar waist girth, the one with greater visceral fat is likely to have an increased health risk relative to the other. The ratio of VAT to SAT has been well correlated to cardiometabolic risk (Kaess et al., 2012). However, expensive medical imaging technologies are required to identify visceral fat, usually involving equipment found only in hospital facilities. However, attempts to predict visceral fat using anthropometry are valuable because they have the potential to use a low cost method to identify higher risk individuals, who may be targeted with appropriate resources and treatment.

Relative girth (as opposed to relative weight) approaches include the well-established waistto-hip ratio (de Koning, et al., 2007) and also waist-to-stature ratio (Ho et al., 2003). While both can claim justification and are straightforward to measure, the question remains as to the likelihood of both numerator and denominator varying to a similar degree. The magnitude of the waist varies by much more than is generally appreciated even in healthy adults approaching $12 \%$ variability in women, and 5\% in men across the 'waist zone' between the 
iliac crest and the $10^{\text {th }}$ rib (Stewart et al., 2010). This not only influences the measurement value but also the accuracy of predicting visceral fat (Bosy-Westphal et al., 2009). This is due, in part, to protocol differences, but also because the shape of the visceral compartment itself varies axially and is prone to under-sampling error (Thomas and Bell, 2003). Waist girth has been shown to have a negative correlation with stature in a large UK sample (Wells, Treleaven and Cole, 2007), which is contrary to assumptions made in a range of anthropometric models that presume taller individuals have larger waists. The reality of using geometric models with girths is that the contained tissue is predicted using a circular model. In reality, the body is not circular in cross section, and the superficial fat depth is not uniform. Challenges to circular models have been levelled using elliptical approaches validated against MRI and have shown them superior to circular models (He et al., 2004). In this, both the total abdominal area, and the visceral compartment area showed very strong predictions from anthropometric models.

Linear measures of skeletal dimensions have helped classify frame size and bodily proportions, but are of limited use in assessing health, because soft tissue is more influential. As such, the brachial, crural and androgyny indices may be more valuable in identifying suitability for sports performance than health concerns. However, other linear dimensions are valuable in a health context. The sagittal abdominal diameter (SAD) at the waist section has received increasing attention over recent years. Several studies have demonstrated its association with visceral fat, and its utility in predicting an adverse metabolic profile (Valsamakis et al., 2004), and incident diabetes (Pajunen et al., 2013). While measurement standardisation is essential to compare results in different studies (due to postural and breathing artefacts), as a single measure, the SAD has much in its favour, either on its own, or in combination with other measurements. 
Other combined measurements have been used to derive composite indices. The conicity index (Valdez, 1991) includes height, weight and waist girth in a theoretical model of opposed truncated cones. The body adiposity index (Bergman et al., 2011) uses body height raised to the power of 1.5 and hip circumference, but subsequent work showed it was no better than BMI, waist or hip girth at predicting fatness (Freedman et al., 2012). The body roundness index considers the eccentricity of the body's height in relation to waist, and was successfully shown to relate to \%fat and \% visceral adipose tissue (Thomas et al., 2013). While this study appears very robust, its assumed circularity of the waist is a limitation, and the assumption that waist circumference should increase with stature is at odds with the aforementioned observations of Wells et al. (2007). Taken together, there are many anthropometric health indices to select from, all of which have their benefits and limitations. Although their applicability may be limited in some groups, especially athletes (Santos et al., 2015), the emergence of newer indices especially those which predict VAT is likely to supplant the over-reliance on some of the more traditional indices which have been used ubiquitously in the past.

\subsection{Applications in sport}

Body composition is only one of many factors (physical, physiological, genetic and psychological) that will determine athletic performance. And while the focus for many coaches and support staff is on adiposity of their athletes, it is important to remember other aspects of body composition, namely the lean tissue components of muscle, bone, ligaments and tendon, also affect sport performance. Many authors have noted that successful athletes in certain sports fit closely to an optimal body composition. While weight-sensitive sports (Ackland et al., 2012) compel the athlete to minimise body mass, other sports (e.g. contact sports) demand robust bodies in a musculoskeletal sense and can generally tolerate athletes with higher proportions of fat (e.g. American football linemen). Furthermore, some events 
(e.g. open water swimming) favour competitors with a degree of adiposity, providing a biomechanical advantage of buoyancy and a physiological benefit of insulation against heat loss.

Regardless of their sporting involvement, athletes appear to be in a perpetual state of body modification; this may be to gain mass, reduce fat or make a weight category for competition. Therefore, it has become increasingly important to monitor the body composition status of athletes throughout the pre- and competition phases of the season. A variety of methods, involving both laboratory-based and field techniques, have been employed for this purpose. Ackland et al. (2012) summarise the current status of these techniques with commentary on the validity, repeatability, assumptions, advantages and limitations of each method.

Meyer et al. (2013) used a 40-item instrument to survey the body composition methods used by sport scientists, and medical and health practitioners world-wide for the assessment of athletes. The data from 159 respondents were stratified according to demographics, sport type and level of competition. While there were different methods favoured in various parts of the world, it was clear that the majority relied upon skinfolds (though there was much variation in both technique standardisation and post-processing of data) as well as DXA. There was also support for Bioelectrical Impedance Analysis (BIA) and Air Displacement Plethysmography (ADP) methods. In terms of measurement frequency, a large cohort of respondents stated that this depended on the individual athlete and which sport they competed in, as well as the general goals of the intervention and phase of the training/competition season. Nevertheless, most athletes who were supported by these survey respondents appeared to be assessed between two to six times per year.

Body composition assessments have also formed an important component of large studies of elite performers at World and Olympic competitions. These studies have the dual purpose of providing contemporary normative data for athlete comparison, as well as to better 
understand the optimal morphology for a particular sport or event in terms of the athletes’ physical and physiological characteristics. The first of these large-scale anthropometric surveys of elite performers began at the Rome Olympics with a study of 137 track and field athletes (Tanner, 1964). Since this important work, anthropometic and other data have been published from the Mexico Olympics (deGaray et al., 1974), Montreal Olympics (Carter, 1982), and Sydney Olympics (Kerr et al., 2007), as well as various World Championships covering many sports.

In one of the most comprehensive studies of aquatics athletes, a team of 33 anthropometrists measures full anthropometric profiles of 919 swimmers, divers, synchronised swimmers and water polo players attending the 1991 World Aquatics Championships in Perth, Western Australia (Carter and Ackland, 1994). For most of the swimming events, $70-80 \%$ of all competitors were assessed, with a similarly high proportion of finalists measured. The data also included 82 divers (80\% of the top 10 competitors), 137 synchronised swimmers (100\% of the top 10 competitors), and 299 water polo players. Selected data for male competitors are shown in Table 6.1. According to Drinkwater and Mazza (1994), long distance (open water) swimmers were significantly fatter (higher SUM6SF) than sprint and 1500 m swimmers this additional adiposity was thought to aid buoyancy and thermal insulation during the open water events. Divers were shorter, lighter and leaner than both swimmers and water polo players. There are significant biomechanical imperatives in the sport of diving that demand such characteristics from elite performers. With this body morphology elite divers are able to minimise their rotational inertia and thereby, maximise somersault and twist velocity whilst in flight. The largest players in water polo are the centre forwards and backs. In these positions, players are subjected to very heavy contact and body checking, and so demand very strong, robust and buoyant bodies. In contrast, the other field position players need to be 
comparatively lighter and faster as they spend much of the time carrying the ball and setting up plays for the centre forward.

Triathletes ( $\mathrm{n}=71$ ) from 11 nations competing in the 1997 Triathlon World Championships were measured on a battery of 28 anthropometric dimensions. A factor analysis (Landers et al., 2000) reduced the number of variables to four (robustness, adiposity segmental lengths and skeletal mass) and these were used in a linear regression to determine which morphological characteristics were important to performance (Table 6.2). With respect to the total elapsed time for the event, and with male and female competitors combined, a regression equation using the adiposity and segmental length factors accounted for $47 \%$ of the variance in triathlon duration. The analysis illustrated the importance of low levels of adiposity and proportionally long limb segments for elite triathletes, especially in regard to total elapsed time, as well as swim time, cycle time and run time treated separately. Higher levels of adiposity hampers run times especially because it increases segmental inertia, diminishes running economy and impairs heat loss. According to Landers et al. (2000) greater adiposity increases the energy demands for an athlete attempting to keep pace with a runner of equal body mass, but with reduced fat mass.

Both male and female light-weight rowers, as we might surmise, have significantly $(\mathrm{p}<0.01)$ reduced stature, body mass and adiposity compared to their open category counterparts (Table 6.3). These data were published for competitors at the Sydney Olympic Games in 2000 (Kerr et al., 2007), where 273 rowers were measured as part of the OZ2000 project. A further analysis compared the 'best' crews (top 7 placings) to the 'rest'. The 'best' open male crews were taller, heavier and more robust than the 'rest', but did not differ in terms of adiposity. For open women rowers, however, lower adiposity levels were characteristic of the 'best' performers. More recently, Schranz et al. (2010) used 3D scanning to capture morphology of elite rowers at two consecutive Australian national championships, and 
compared these to a control group of the general population. This insightful study was the first if its kind with body scanning, and was able to establish the effect size for a range of morphological variables which differed between rowers and controls such as cross sectional areas, surface areas and segment volumes, which are not available using conventional anthropometry. As with the Olympic study, these data provide valuable information for coaches and support staff when selecting, developing and monitoring elite level crews. While there are clear examples of absolute body size being advantageous, most obviously in basketball players and racing jockeys, there are subtler examples where the body's skeletal proportions may be influential. Short track speed skating favours the short limb length, low centre of mass and rapid power development. The dominance of oriental nations such as Korea and Japan illustrate this point. Skeletal proportions are not 'trainable', therefore athletes can be expected to self-select into sports in which they are likely to excel (Stewart et al., 2011). For example, speed and endurance runners have greater crural indices (tibia:femur length ratio) which reduces inertial resistance. This concept extends to the identification of sporting talent, which has historically targeted exceptionally tall young rowers, with the potential to have long limbs and greater leverage. Onto this skeletal framework soft tissues of muscle and fat respond to the training regimes and can be optimised for gaining and losing body weight, in a periodised training cycle.

The assessment and monitoring of body composition, using both anthropometric and other techniques, has become fundamental in the preparation of elite sporting competitors. As athletes strive to gain weight, lose weight and make weight for competition, they are invariably subject to health risks due to some extreme practices. This is especially poignant for those competing in weight-sensitive sports - those sports in which high body weight restricts performance (gravitational sports), or where the athlete must meet a weight category restriction (weight-class sports), or in which the aesthetics of the performance contributes to 
the outcome (aesthetic sports). In a recent paper, Sundgot-Borgen et al. (2013) addressed some of the behaviours and health risks associated with these weight-sensitive sports and provided guidelines for athletes, coaches, support staff and administrators for recognising and preventing eating disorders. The authors also provide recommendations for sports administrators to consider rule modifications in their sport with a view to minimising or eliminating some of the unhealthy behaviours and practices that these rules promote.

\subsection{Applications in ergonomics}

Ergonomic applications of body dimensions relate the human shape and posture to function in working and living environments. The interface between human form and functional activity is at the very core of ergonomics, and influences a range of applications and agendas related to risk, safety, comfort and productivity. The science of ergonomics encompasses a range of scientific disciplines - including physiology, biomechanics, human behaviour and others, all of which relate directly or indirectly to health and function. Many ergonomics challenges relate to such factors as vision, cognition, fatigue and work capacity in complex ways, while others relate to body size in a more direct way.

Indigenous populations vary profoundly in their size and proportions, with the implication that size standards in one country may not be applicable in another. Genetic hybridization and secular trends for increase in stature (Cole, 2002) have both influenced current, observed body size and bodily proportions, with implications for injury risk such as when operating machinery. The rising prevalence of global obesity (WHO, 2000) has arguably had an additive effect which has been more rapid and profound by affecting posture and locomotion (Wearing et al., 2006), and compromising movement and effective work in restricted space, with consequences for musculo-skeletal health (Gallagher, 2005).

Body size is also not the same between different professions within a country, and evidence exists that certain professions are associated with larger individuals, including truck drivers 
(Guan and Hsaio, 2012) and firefighters (Hsaio et al., 2014). This has implication for extrapolating national size data to certain at-risk groups. As an example, consider the 67,000 UK offshore workers who are transported by helicopter to and from their work on installations in the North Sea. It was well established from a previous survey that UK offshore workers were heavier than UK norms (Light and Dingwall, 1985), but this disparity in body weight has trebled since that time, during which the average male worker's weight increased by 19\%. A subsequent study sought to quantify a large number of dimensions in relation to this trend for increased size (Ledingham et al., 2015).

In a recent safety review of helicopter operations, the UK Civil Aviation Authority altered seating rules to facilitate compatibility between passenger body size and the window diameter on the corresponding seat row. A recent study (Stewart et al., 2015), using a sub-sample of 404 offshore workers sampled across the entire weight spectrum, involved egressing through a window frame representing the minimum allowable size. Unsurprisingly, smaller individuals were more likely to succeed than their larger counterparts. Individual measurement dimensions obtained from 3D scanning were subjected to binary logistic regression to determine the optimal predictive test. Scrutiny of the effect size for the dimensional difference between those who passed and failed the egress task revealed any one of a number of candidate measurements (see figure 6.2) could be used to inform an intelligent seating policy, although some of the measurements would not be practical to undertake for the industry. However, some false positives (large individuals succeeding) and false negatives (smaller individuals failing) suggest that body size is not the only predictive variable and that other factors such as flexibility and technique could also be influential.

\subsection{Conclusion}


What is the current state of anthropometry within the context of performance and health? Some would argue that the emergence of new techniques which are becoming increasingly affordable and portable, especially ultrasound and 3D scanning, could spell the end of the road for traditional anthropometry. Ethnic groups, children and the elderly, together with participants from virtually every sport have already been characterised by anthropometry. While some studies of this nature may not be repeated, it is worth recognising that very few studies with physical activity and performance or the wider realm of epidemiology do not involve some anthropometric measurements. Although it is hard to understand why the advances in standardisation in traditional anthropometry were so long in coming, it is easy to recognise their benefits. The time is now ripe for equivalent standardisation of 3D anthropometry (a young and rapidly evolving science by comparison), which will enable robust quality assurance of data, and an audit trail of competencies seen across other body composition disciplines. This will be essential if we are to create integrated composite models using a combination of methods, which could use a digital 3D template enriched by composition data located in xyz space. Such a 'holy grail' could be expanded to include large data repositories and sharing arrangements which would help us understand much finer morphological detail due to enhanced power to detect small differences, such as the intra and inter-personal gradient in physique with sports performance standard, or shape change with disease progression with ageing or muscle wasting.

However, we are not there yet, despite the aspiration of some, and the technological competence of many. First, as anthropometrists and practitioners of body composition we need a more complete dialogue and to agree a common language on definitions, standards and protocols. Second, we need to define our aims collectively and build a path and structure which will help us towards achieving them. Neither of these tasks is straightforward, and both go well beyond the job descriptions of experts in the field. However, history will relate 
the pivotal role of key researchers who have defied the odds in encouraging entire disciplines to move in a certain direction. Individuals might need persuading that such efforts are worthwhile, because of the political hurdles in the path of international application and implementation. While there will always be differences on which path it is best to take, thanks to modern communication media, never before has such international dialogue been as straightforward. The prominence of anthropometric measurement within wider field of body composition throughout history will only be maintained or enhanced if such exchange of ideas embraces these new concepts. However, with the enthusiasm of its practitioners and the exciting developments anticipated, anthropometry may expect a promising future.

\section{References}

Ackland, T. R., T. G. Lohman, J. Sundgot-Borgen, et al. 2012. Current status of body composition assessment in sport. Sports Med. 42:227-249.

Adams, J., M. Mottola, K.M., Bagnell and K. D. McFadden. 1982. Total body fat content in a group of professional football players. Can. J. Appl. Sport Sci. 7:36-40.

Bergman, R. N., D. Stefanovski, T. A. Buchanan, et al. 2011. A better index of body adiposity. Obesity (Silver Spring) 19:1083-1989.

Bjorntorp, P. 1997. Body fat distribution, insulin resistance, and metabolic diseases. Nutrition, 13:975-803.

Bosy-Westphal, A., C-A., Booke, T. Blöcker, et al. 2009. Measurement site for waist circumference affects its accuracy as an index of visceral and abdominal subcutaneous fat in a Caucasian population. J. Nutr.140:954-961.

British Standards Institute 2010. 3-D scanning methologies for internationally compatible anthropometric databases. BS EN ISO 20685:2010. 
Carin-Levy, G., C. A Greig, S. J. Lewis, A., Stewart, A. Young, and G. E. Mead. 2008. The effect of different positions on anthropometric measurements and derived estimates of body composition. Int.J. Body Comp. Res. 6:17-20.

Carter, J. E. L. and B. H. Heath., 1990. Somatotyping - Development and Applications. Cambridge: Cambridge University Press.

Carter, J. E. L. 1985. Morphological factors limiting human performance. In The limits of human performance The American Academy of Physical Education Papers, No. 18. ed. H. M. Eckert and D. H. Clarke, 106-17. Champaign (IL): Human Kinetics.

Carter, J. E. L. and T. R. Ackland. 1994. Kinanthropometry in Aquatic Sports. Champaign IL: Human Kinetics.

Carter, J. E. L. 1982. Physical Structure of Olympic Athletes. Part 1. The Montreal Olympic Games Anthropological Study. Basel, Switzerland: Karger.

Clarys, J. P., A. D. Martin, D. T. Drinkwater, and M. J. Marfell-Jones, M.J. 1987. The skinfold: myth and reality. J. Sports Sci. 5:3-33.

Cole, T. 2002. The secular trend in human physical growth: a biological view. Econ. Hum. Biol. 1:161-168.

Daniell, N., T. Olds and G. Tomkinson. 2010. The importance of site location in girth measurements. J. Sports Sci. 28:751-757.

deGaray, A. L., L. Levine and J. E. L. Carter, 1974. Genetic and Anthropological Studies of Olympic Athletes. New York: Academic Press.

de Koning, L., A. T. Merchant, J. Pogue and S.S. Anand. 2007. Waist circumference and waist-to-hip ratio as predictors of cardiovascular events: meta-regression analysis of prospective studies. Eur. Heart J. 28:850-856.

Drinkwater D. T. and W. D. Ross. 1980. Anthropometric Fractionation of Body Mass. In Kinanthropometry II , ed. W. Ostyn, G. Beunen and J. Simons 177-188. Baltimore: 
University Park Press.

Drinkwater, D. T. and J. C. Mazza, 1994. Body composition. In Kinanthropometry in Aquatic Sports, ed. J. E. L. Carter and T. R. Ackland, 102-137. Champaign IL: Human Kinetics. Durnin, J. V. G. A. and J. Womersley. 1974. Body fat assessed from total body density and its estimation from skinfold thickness: measurements on 481 men and women aged from 16 to 72 years. Brit. J. Nutr. 32:77-97.

Evans, E. M., D. A. Rowe, M. M. Misic, B. M., Prior and S. A. Arngrimsson. 2005. Skinfold prediction equation for athletes developed using a four-compartment model. Med. \& Sci. in Sports \& Exerc. 37:2006-2011.

Freedman, D.S., J. Thornton, F. X. Pi-Sunyer et al. 2012. The body adiposity index (hip circumference/height(1.5)) is not a more accurate measure of adiposity than is BMI, waist circumference, or hip circumference. Obesity (Silver Spring) 20:2438-2444.

Gallagher, D., S. B. Heymsfield, M. Heo, S. A. Jebb, P. R. Murgatroyd and Y. Sakamoto. 2000. Healthy percentage fat ranges: an approach for developing guidelines based on body mass index. Am. J. Clin. Nutr. 72:694-701.

Gallacher, S. 2005. Physical limitations and musculoskeletal complaints associated with work in unusual or restricted postures: A literature review. J. Safety Res. 35:51-61.

Guan, J. and H. Hsaio. 2012. U.S. Truck driver anthropometric study and multivariate anthropometric models for cab designs. Hum.Factors 54:849-871.

He, Q., E. S. Engelson, J. Wang et al. 2004. Validation of an elliptical anthropometric model to estimate visceral compartment area. Obes. Res. 12:250-257.

Himes, J. H., A. F. Roche, and R. M. Siervogel. 1979. Compressibility of skinfolds and the measurement of subcutaneous fatness. Am. J. Clin. Nutr. 32:1734-1740.

Ho, S-Y., T-H. Lam and E. D. Janus. for the Hong Kong cardiovascular risk factor prevalence study steering committee. 2003. Waist to stature ratio is more strongly 
associated with cardiovascular risk factors than other simple anthropometric indices. Ann. Epidemiol. 13:683-691.

Hsaio, H., J. Whitestone, T. Kau, R. Whisler, G., Routley and M. Wilbur. 2014. Sizing firefighters: method and implications. Hum Factors 56:873-910.

Hume, P. and M. Marfell-Jones. 2008. The importance of accurate site location for skinfold measurement J. Sports Sci. 26:1333-1340.

Jackson, A. S. and Pollock, M. L. 1978. Generalized equations for predicting body density of men. Brit. J. Nutr. 40:497-504.

Jackson, A. S., M. L. Pollock and A. Ward. 1980. Generalized equations for predicting body density of women. Med. Sci. Sports Exerc. 12:175-182.

Kaess, B. M., A. Pedley, J. M. Massaro, J. Murabito, U. Hoffman, and C. S. Fox. 2012. Diabetologia 55:2622-2630.

Kerr, D. A., T. R Ackland, W. D. Ross, K. Norton and P. Hume, P. 2007. Olympic lightweight and open rowers possess distinctive physical and proportionality characteristics. J. Sports Sci. 25:43-53.

Kershaw, E. E. and J. S. Flier. 2004. Adipose Tissue as an Endocrine Organ. J. Clin. Endocrinol. Metab. 89:2548-2556 .

Kuk, J. L., P. T. Katzmarzyk, N. Z. Nichaman, T. S. Church, S. N. Blair and R. Ross. 2006. Visceral fat is an independent predictor of all-cause mortality in men. Obesity (Silver Spring) 14:336-341.

Kupke, T. and T. Olds. 2008. Towards a generalised anthropometric language. In Kinanthropometry IX. Proceedings of the $10^{\text {th }}$ International Conference of the International Society for the Advancement of Kinanthropometry ed. M. Marfell-Jones and T. Olds, 213-230. London: Routledge. 
Landers, G. J., B. A. Blanksby, T. R. Ackland and D. Smith. 2000. Morphology and performance of world championship triathletes. Ann.Hum. Biol. 27:387-400.

Ledingham, R., G. Alekandrova, M. Lamb and A. Stewart. 2015. Size and Shape of the UK Offshore Workforce 2014: A 3D scanning survey. Robert Gordon University. ISBN 978-1-907349-10-2.

Light, I. M. and R. H. M. Dingwall. 1985. Basic anthropometry of 419 offshore workers (1984). Offshore Survival Centre, Robert Gordon’s Institute of Technology, Aberdeen, UK.

Lohman, T. G. 1992. Advances in body composition assessment. Current issues in exercise science series monograph no.3. Champaign, IL: Human Kinetics.

Lohman, T. G., A. F. Roche and R. Martorell. 1988. Anthropometric standardization reference manual. Champaign, IL: Human Kinetics.

Martin, A. D. 1991. Anthropometric assessment of bone mineral. In Anthropometric Assessment of Nutritional Status ed. J. Himes, 185-196. New York: Wiley-Liss.

Martin, A. D., Drinkwater, D. T., Clarys, J. P. and Ross, W.D. 1986. The inconstancy of the fatfree mass: a reappraisal with implications for densitometry. In Kinanthropometry III ed. T. Reilly, J. Watkins and J. Borms, 92-97. London: E. \& F. N. Spon.

Martin, A. D., L. F. Spenst, D. T. Drinkwater and J. P. Clarys. 1990. Anthropometric estimation of muscle mass in men. Med. Sci. Sports Exerc. 22:729-733.

Mateigka, J. 1921. The testing of physical efficiency. Am. J. Phys. Anthropol. 4:223-230.

Meyer, N.L., Sundgot-Borgen, J., Lohman et al. 2013. Body composition for health and performance: A survey of the ad hoc research working group on body composition health and performance, under the auspices of the IOC Medical Commission. Brit. J. Sports Med. 47:1044-1053. 
Müller, W. 2009. Towards research-based approaches for solving body composition problems in sports: ski jumping as a heuristic example. Brit. J. Sports Med. 43:1013-1019

Müller, W., M. Horn, A. Fürhapter-Rieger et al. 2013a.Body Composition in Sport: a comparison of novel ultrasound imaging technique to measure subcutaneous fat tissue compared with skinfold measurement. Brit. J. Sports Med. 47:1028-1035,

Müller, W., M. Horn, A. Fürhapter-Rieger et al. 2013b. Body Composition in Sport: Interobserver reliability of a novel ultrasound measure of subcutaneous fat tissue. Brit. $J$. Sports Med. 47:1036-1043.

Müller, W., T. G. Lohman, A. D. Stewart et al. 2016. Subcutaneous fat patterning in athletes: selection of appropriate sites and standardisation of a novel ultrasound measurement technique. Brit. J. Sports Med. 50:45-54.

Norton, K. and T. Olds. 1996. Anthropometrica. Sydney: University of New South Wales Press.

Olds, T. 2004. The rise and fall of anthropometry. J. Sports Sci. 22, 319-320.

Olds, T., N. Daniell, J. Petkov and A. D. Stewart. 2013. Somatotyping using 3D anthropometry: a cluster analysis. J. Sports Sci. 31:936-944.

Olds, T., G. Tomkinson, M. Rogers, T., Kupke, L., Lowe, L. and N. Daniell. 2004. 3D Anthropometry ADAPT Landmarking Manual. Adelaide SA: University of South Australia.

Pajunen, P., M. Heliövaara, H. Rissanen, A. Reunanen, M. A. Laaksonen and P. Knekt. 2013. Sagittal abdominal diameter as a new predictor if incident diabetes. Diabetes Care, 36:283-288.

Perini, T. A., G. L. de Oliveira, J. S. Ornellas, and F. P. de Oliveira. 2005. Technical error of measurement in anthropometry. Rev. Bras.Med. Esporte 11:86-90. 
Robinette, K. M., S. Blackwell, M. Daanen et al. 2002. Civilian American and European surface anthropometry resource (CAESAR) Final Report, Vol 1: Summary. United States Air Force Research Laboratory, Wright-Patterson Air Force Base, Ohio, U.S.

Santos, D.A., A. M. Silva, C. N. Matias et al. 2015. Utility of novel indices in predicting fat mass in elite athletes. Nutrition 31:948-954.

Schranz, N., G. Tomkinson, T. Olds, and N. Daniell. 2010. Three-dimensional anthropometric analysis: Differences between elite Australian rowers and the general population. $J$. Sports Sci.28:459-469.

Sinning, W. E., D. G. Dolny, K. D. Little et al 1985. Validity of “generalised” equations for body composition analysis in male athletes. Med. Sci. Sports Exerc. 17:124-130.

Stewart, A., R. Ledingham, G. Furnace, N. Schranz and A. Nevill. 2015. The ability of UK offshore workers of different body size and shape to egress through a restricted window space. Appl. Ergon. (In press corrected proof http://dx.doi.org/10.1016/j.apergo.2015.11.005).

Stewart, A., M. Marfell-Jones, T. Olds and H. de Ridder. 2011. International Standards for Anthropometric Assessment. International Society for the Advancement of Kinanthropometry, Lower Hutt, New Zealand.

Stewart, A. D. 2010. Kinanthropometry and Body Composition: a natural home for 3D photonic scanning. J. Sports Sci.28, 455-457.

Stewart, A. D. and W. J. Hannan. 2000. Prediction of fat and fat-free mass in male athletes using dual X-ray absorptiometry as the reference method. J. Sports Sci.18:263-274.

Stewart, A. D., A. M. Nevill, R. Stephen and J. Young. 2010. Waist size and shape assessed by 3D photonic scanning. Int. J. Body Comp. Res. 8:123-130. 
Stewart, A.D., A. Stewart and D. Reid. 2002. Correcting calf circumference discriminates the incidence of falling but not bone quality by broadband ultrasound attenuation in elderly female subjects. Bone, 31:195-198.

Stewart, A.D., P. J. Benson, T. Olds, M. Marfell-Jones, A. MacSween and A. M. Nevill. 2011. Self-selection of athletes into sports via skeletal ratios. J. Contemporary Athletics 5:153-167.

Sundgot-Borgen, J., N. L. Meyer, T. G. Lohman et al. 2013. How to minimize risks for athletes in weight-sensitive sports. Brit. J. Sports Med. 47:1012-1022.

Tanner, J.M. 1964. The physique of the Olympic Athlete. London: George Allen \& Unwin Ltd.

Thomas, D. M., C. Bredlau, A. Bosy-Westphal et al. 2013. Relationships between body roundness with body fat and visceral adipose tissue emerging from a new geometrical model. Obesity (Silver Spring), 21:2264-2271.

Thomas, E. L. and J. D. Bell. 2003. Influence of undersampling on magnetic resonance imaging measurements of intra-abdominal adipose tissue. Int. J. Obes. 27:211-218.

Tyrrell, A. R., T. Reilly. and J. D. G. Troup. 1985. Circadian variation in stature and the effects of spinal loading. Spine 10:161-164.

Valdez, R. 1991. A simple model-based index of abdominal adiposity. J. Clin. Epidemiol. 9:955-956.

Valsamakis, G., R. Chetty, A. Anwar, A. K. Banerjee, A. Barnett and S. Kumar. 2004. Association of simple anthropometric measures of obesity with visceral fat and the metabolic syndrome. Diabet. Med. 21:1339-1448.

Wang, J., D. Gallagher, J. C. Thornton, W. Yu, M. Horlick and F. X. Pi-Sunyer. 2006. Validation of a 3-dimensional photonic scanner for the measurement of body volumes, dimensions, and percentage body fat. Am. J. Clin. Nutr. 83:809-816. 
Wearing, S. C., E. M. Henning, N. M., Byrne, J. R. Steele and A. P. Hills. 2006. The biomechanics of restricted movement in adult obesity. Obes. Rev. 7:13-24.

Wells, J. C., P. Treleaven and T. J. Cole. 2007. BMI compared with 3-dimensional body shape: the UK National Sizing Survey. Am. J. Clin. Nutr, 85:419-425.

World Health Organization. 2000. Obesity: preventing and managing the global epidemic. Report of a WHO Consultation. WHO Technical Report Series 894. Geneva: World Health Organization. 
Table 6.1. Body composition of male aquatic athletes competing at the 1991 World Championships.

\begin{tabular}{|c|c|c|c|c|c|c|}
\hline Event & $\mathrm{n}$ & $\begin{array}{l}\text { Stature } \\
(\mathrm{cm})\end{array}$ & $\begin{array}{l}\text { Body mass } \\
(\mathrm{kg})\end{array}$ & $\begin{array}{c}\text { SUM6SF }^{1} \\
(\mathrm{~mm})\end{array}$ & $\begin{array}{c}\text { Muscle mass }{ }^{2} \\
(\mathrm{~kg})\end{array}$ & $\begin{array}{c}\text { Skeletal mass }{ }^{3} \\
(\mathrm{~kg})\end{array}$ \\
\hline \multicolumn{7}{|c|}{ Freestyle Swimming } \\
\hline $50 \& 100 \mathrm{~m}$ & 47 & 186.4 & 79.8 & 44.0 & 57.8 & 13.3 \\
\hline $200 \& 400 \mathrm{~m}$ & 34 & 185.2 & 79.1 & 50.4 & 57.0 & 13.0 \\
\hline $1500 \mathrm{~m}$ & 10 & 183.1 & 74.3 & 41.8 & 56.1 & 13.9 \\
\hline Long distance & 13 & 179.6 & 78.1 & 60.3 & 56.3 & 13.0 \\
\hline Diving & 43 & 170.9 & 66.7 & 45.9 & 58.6 & 12.9 \\
\hline \multicolumn{7}{|l|}{ Waterpolo } \\
\hline Goalkeepers & 30 & 189.1 & 86.2 & 60.9 & 56.6 & 13.1 \\
\hline Centre backs & 25 & 189.2 & 90.2 & 65.0 & 56.9 & 12.7 \\
\hline Centre forwards & 40 & 188.8 & 91.4 & 68.3 & 55.5 & 12.6 \\
\hline Others & 95 & 184.0 & 82.7 & 59.9 & 55.8 & 12.8 \\
\hline
\end{tabular}

${ }^{1}$ Sum of triceps, subscapular, abdominal, supraspinale, front thigh and medial calf skinfolds

${ }^{2}$ Estimated muscle mass calculated from anthropometry according to Martin et al. (1990)

${ }^{3}$ Estimated skeletal mass calculated from anthropometry according to Martin et al. (1991)

Table 6.2. Factor B weights and regression coefficients for male and female World Championship triathletes. Where $\mathrm{x} 1$ = adiposity; $\mathrm{x} 2$ = segmental lengths.

\begin{tabular}{cccccc}
\hline $\begin{array}{c}\text { Dependent } \\
\text { variable (y) }\end{array}$ & B0 & B1 & B2 & $\mathrm{R}^{2}$ & $\begin{array}{c}\mathrm{F} \\
(*=\text { sig }<<0.01)\end{array}$ \\
\hline Total time (s) & 7434.9 & 324.8 & -180.2 & 0.688 & $29.20^{*}$ \\
Swim time (s) & 1232.6 & 31.9 & -44.3 & 0.549 & $17.10^{*}$ \\
Cycle time (s) & 3892.6 & 149.5 & -99.9 & 0.659 & $24.88 *$ \\
Run time (s) & 2201.0 & 146.2 & -- & 0.618 & $40.84 *$ \\
\hline
\end{tabular}

Note: general format for the multiple regression equation is: $\mathrm{y}=\mathrm{B} 0+(\mathrm{B} 1 \cdot \mathrm{x} 1)+(\mathrm{B} 2 \cdot \mathrm{x} 2)+\mathrm{Bn} \cdot \mathrm{xn})$

Table 6.3. Anthropometric characteristics of Olympic lightweight and open-class rowers.

\begin{tabular}{|l|c|c|c|c|c|}
\hline \multirow{2}{*}{ Variable } & \multicolumn{2}{|c|}{ Male Rowers } & & \multicolumn{2}{c|}{ Female Rowers } \\
\cline { 2 - 5 } & $\begin{array}{c}\text { Lightweight } \\
(\mathrm{n}=50)\end{array}$ & $\begin{array}{c}\text { Open-class } \\
(\mathrm{n}=140)\end{array}$ & & $\begin{array}{c}\text { Lightweight } \\
(\mathrm{n}=14)\end{array}$ & $\begin{array}{c}\text { Open-class } \\
(\mathrm{n}=69)\end{array}$ \\
\hline Age $(\mathrm{y})$ & 27.1 & 26.4 & & 26.0 & 27.8 \\
\hline Stature $(\mathrm{m})$ & 1.82 & 1.94 & & 1.69 & 1.81 \\
\hline Sitting height $(\mathrm{m})$ & 0.95 & 0.99 & & 0.89 & 0.94 \\
\hline Body mass $(\mathrm{kg})$ & 72.5 & 94.3 & & 58.5 & 76.6 \\
\hline SUM8SF $^{1}$ & 44.7 & 65.3 & & 59.7 & 89.0 \\
\hline
\end{tabular}

${ }^{1}$ Sum of triceps, biceps, iliac crest, subscapular, supraspinale, abdominal, front thigh and medial calf. 


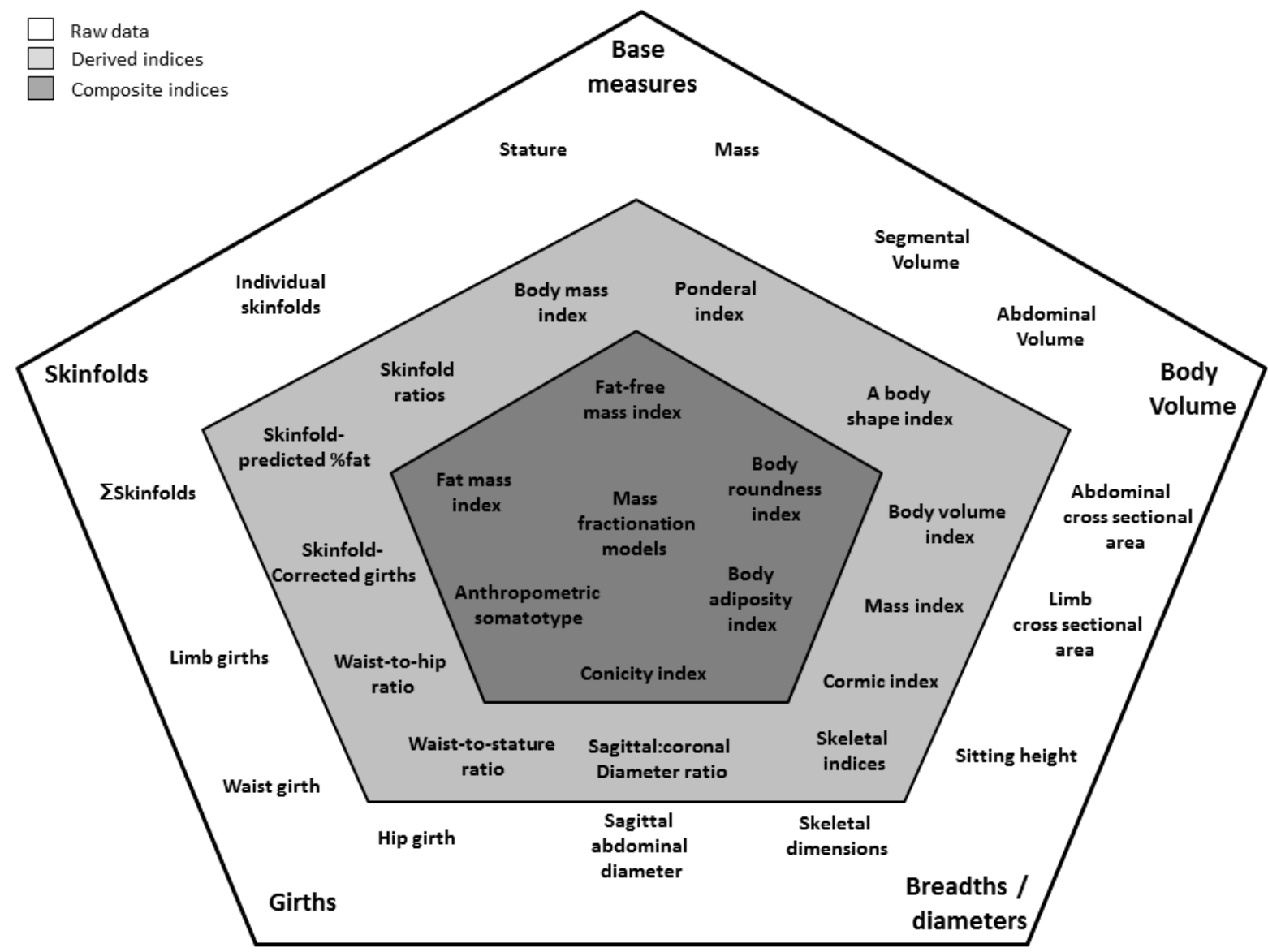

Figure 6.1 Schematic framework of anthropometric measurements, derived indices and composite indices to describe anatomical factors. 


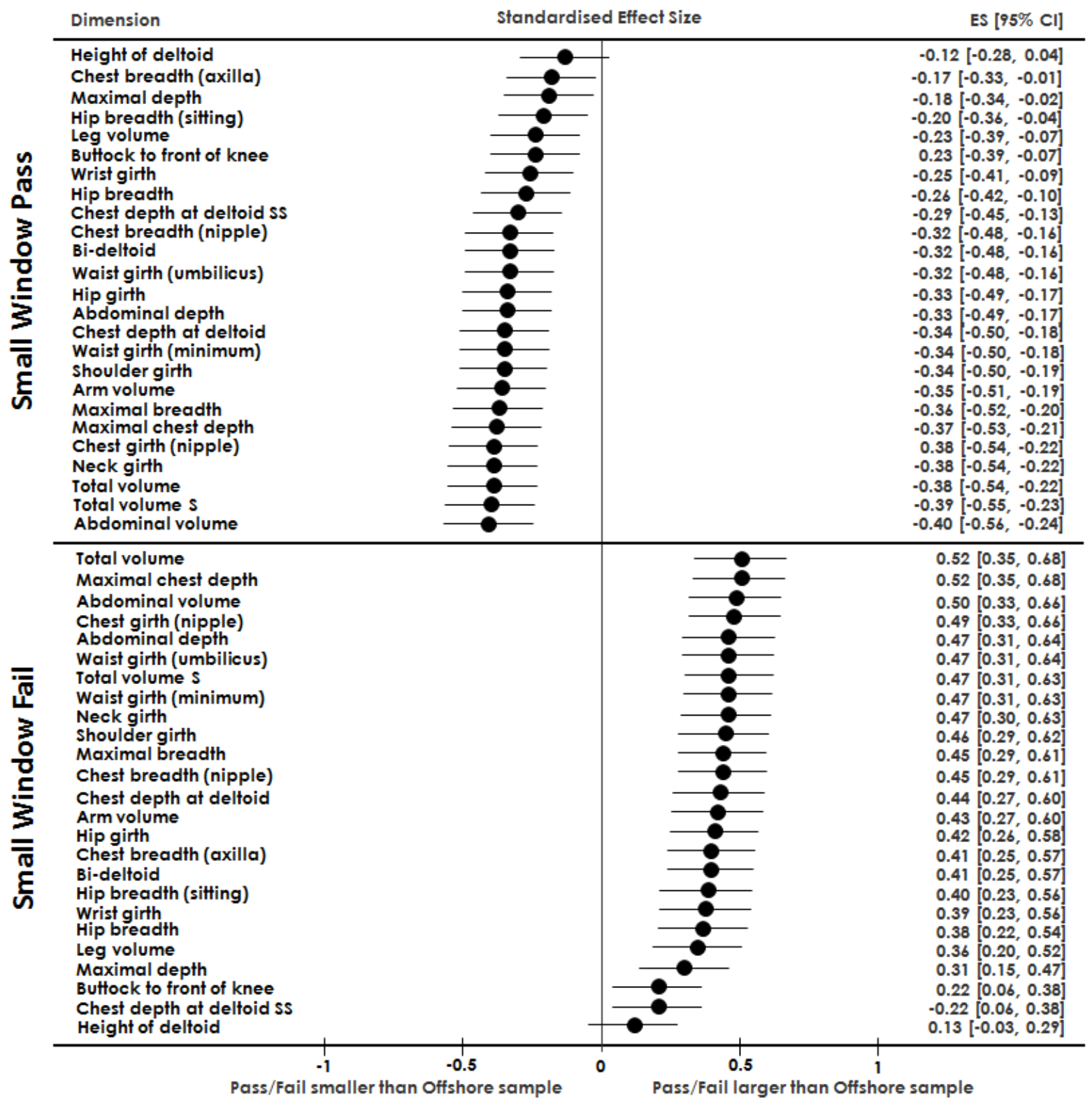

Figure 6.2. Comparison of the effect size of body dimensions between those passing and those failing a helicopter window egress test (Reprinted from Stewart et al. 2015), with permission from Elsevier. 